\title{
A SURVEY ON COMPONENTS OF VIRTUAL 'UMRAH APPLICATION
}

\author{
Normala Rahim, Nazirah Abd Hamid, Wan Malini Wan Isa, Siti Dhalila Mohd \\ Satar and Azilawati Rozaimee \\ Faculty of Informatics and Computing (FIK) \\ University of Sultan Zainal Abidin (UniSZA), \\ 21300 Gong Badak, Terengganu, Malaysia
}

\begin{abstract}
This paper attempts to present insight of components in Virtual 'Umrah application. This interactive application consists of five components namely content, virtual reality (VR) technology, multimedia elements, user profile and usability evaluation. Virtual reality technique in this application enables to provide a realistic experience for the users in performing 'Umrah. The methodology that has been adopted to develop this application is User-Centered design model (UCD) that focuses on involvement of users in every phase. These components are hopefully can be implemented as guidelines to the others to develop a virtual environment.
\end{abstract}

\section{KEYWORDS}

Multimedia Elements, User-Centered Design, Usability, Virtual Reality and 'Umrah

\section{INTRODUCTION}

The development of digital world has dramatically changed the ways of organizations, educational entities, research institutes and medical institutions conduct their tasks included business, teaching and learning and also training. Furthermore, the technology is transforming the way any organizations interact with their customers. This research tries to take advantages of that situation by combining multimedia elements with virtual reality technology that will encourage users to actively interact with the application in gaining experience and knowledge.

Virtual reality technology has been commonly used in some areas such as in training simulators, games and medical procedures. However this technology has not been well adopted in Hajj or 'Umrah environment. Our research will seek to enhance users understanding on the concepts and requirements pertaining to Hajj or 'Umrah through the implementation of virtual reality.

At the moment, there are many applications available for Hajj or 'Umrah such as M-umrah and 'Navigasi Umrah Maya'. However, these existing applications unable to adequately addressed important information effectively to the users particularly to the pilgrims [1] [2]. This situation may lead to the misunderstanding on 'Umrah activities and rituals. Moreover the existing applications on training of 'Umrah still rely very much on conventional method such as plain text in delivering the knowledge. Most of the available applications are slacking in integrating any multimedia elements or Virtual Reality capabilities. 
The International Journal of Multimedia \& Its Applications (IJMA) Vol.5, No.5, October 2013

These drawbacks serve as the main motivation for us to produce an interactive application called Virtual 'Umrah. The proposed Virtual 'Umrah will base on the simulation through the technique calls as Virtual Reality. Virtual Reality technique in this application will be able to provide the most realistic experience for the users in performing 'Umrah. The methodology that has been adopted to develop this application is User-Centered Design model (UCD) that focuses on involvement of users in every phase.

\section{RELATED WORKS}

The growths of Information and Communication Technologies (ICT) have caused the volume of learning materials in recent years accelerated dramatically. The sudden surge in the amount of the learning materials has initiated to issues regarding the quality of the existed materials. According to Yusoff et al. [2], there are many existing 'Umrah supplementary learning materials in the market but has failed to provide clear understanding to users, particularly the pilgrims. The authors also have stated that the existing learning materials styles of 'Umrah should be altered into an active learning method to offer a better learning aid to the users [2]. Similarly, Hameed has suggested an educational model of Hajj to provide trusted and comprehensive information on the whole process of Hajj and related activities. Apart of that, from his literature reviews and surveys, he discovered that very limited learning materials pertaining 'Umrah in the market [1].

Besides the issue of quality learning materials, the issue regarding the methods of delivering is also a concern. Existing software only uses conventional methods such as texts and pictures in delivering knowledge about 'Umrah. This situation leads to misunderstanding among the pilgrims especially on 'Umrah activities. There are lots of researches that have been conducted to overcome the limitations. Yusoff et al. [2] in his research has recommended of using multimedia authoring technique that integrated with virtual environments and persuasive technology design. Moreover this proposed technique can offer supplementary learning materials that support active learning and self-directed learning environment. Other than that, the authors also have developed a 3D simulation that uses virtual environment technique called 3D Tawaf. This 3D Tawaf simulation acts as an interactive training method for Hajj education in Malaysia by providing user a scene of Tawaf (one of Hajj pilgrimage rituals). The main contributions of virtual environment technique are to provide flexible control for the users and deliver a user-friendly and flexible environment [2]. Anita et al. (2010) has also proposed virtual environment technique to simulate real environment in performing one of the rituals in Hajj. The virtual reality is been implemented in assisting the pilgrims in their training of Sa'i. The Sa'i training is developed in $3 \mathrm{D}$ environment using an avatar so that Hajj trainers can be totally immersed into real environment through the simulation and thus increasing their understanding on the ritual of Sa'i effortlessly [3].

Many existing websites regarding Hajj and 'Umrah usually have neglected few important aspects in designing a website such as useful and usability concepts. According to Al-Aama (2008) to accommodate those problems, the author has introduced six steps of methodology in designing a website that are based on user-centered approach. This approach focuses on the users or pilgrims. It starts by identifying the requirements, followed by building of a prototype, then executing usability testing and lastly is the process of evaluating the design. This proposed methodology can produce a usable website for a pilgrim to find the intended information easily and smoothly [4]. 
The International Journal of Multimedia \& Its Applications (IJMA) Vol.5, No.5, October 2013

\section{COMPOnENTs of Virtual 'UmRah Application}

This interactive application consists of five components namely content, virtual reality (VR) technology, multimedia elements, user profile and usability evaluation. These components are hopefully can be implemented as guidelines to the others to develop a virtual environment.

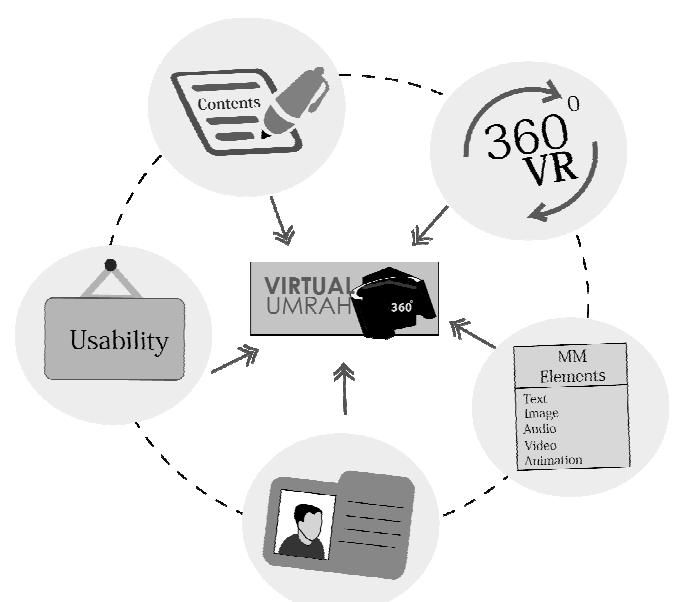

Figure 1. Virtual 'Umrah components

\subsection{Content}

Content is an important component that must be considered when developing Virtual 'Umrah because the contents will be able to provide accurate and precise information on rituals and activities in pertaining 'Umrah. In Virtual 'Umrah application, the contents are made of four parts namely introduction to 'Umrah, preparation for performing 'Umrah, the pillars of 'Umrah and wajib 'Umrah.

\subsubsection{Introduction of 'Umrah}

According to Jamalluddin, 'Umrah is obligatory to perform at least once in a lifetime [5]. This section explains basic information on 'Umrah such as the meaning and decree of 'Umrah, advantages of 'Umrah and time to perform 'Umrah. These information are important to be understood by pilgrims before performing 'Umrah to avoid any misunderstanding and problems during performing 'Umrah.

\subsubsection{Preparation for perform 'Umrah}

This section discusses the information that is very needed before performing 'Umrah. The pilgrims should learn about travelling prayer and manners, ihram, miqat, and sunnatul practice before, during and after performing 'Umrah. Moreover, pilgrims should possess knowledge about history of Mecca and Medina and manners during visit this two Holy City.

\subsubsection{The Pillars of 'Umrah}

The main content of this application is the pillars of 'Umrah. The pillars of 'Umrah consist of five principles as in Figure 2. These pillars must be completed by pilgrims when performing 
'Umrah and if they unable to complete it either intentionally or not, their 'Umrah is not valid. In addition, the application also deliberates on the conditions of sunnatul and du'a.

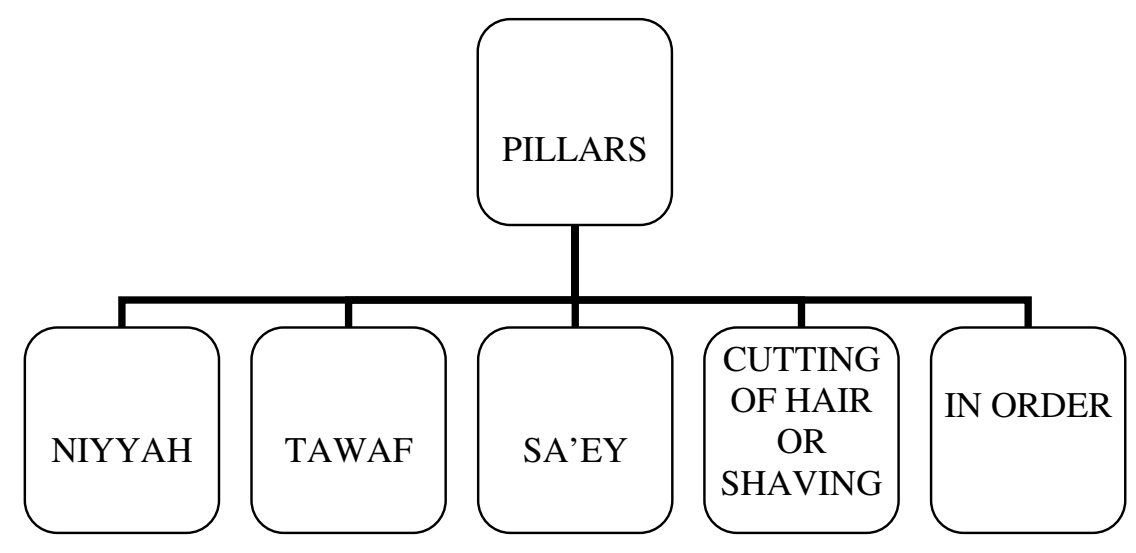

Figure 2. The pillars of 'Umrah

The principles of the pillars of 'Umrah are:

a) Niyyah: Express intention to perform 'Umrah. It can be categorized into three namely normal niyyah, conditional niyyah and badal niyyah (on behalf of other person).

b) Tawaf: Circling around the Ka'bah for seven rounds with certain conditions. The pilgrims must walk start from the Black Stone by keeping the Ka'bah on the left in clockwise manner and end at Black Stone.

c) Sa'ey: Walk back and forth between the hill of Safa and Marwah for seven times. There are certain conditions must be obeyed during Sa'ey.

d) Cutting of hair or shaving: After completion of sa'ey, pilgrims should cut off at least three pieces of their hair.

\subsubsection{Wajib ‘Umrah}

Wajib 'Umrah consists of conditions that must be done during 'Umrah. If pilgrims fail to perform one of these conditions, the 'Umrah is remained valid but the pilgrims have to be fined. If the pilgrims deliberately leave one of the conditions, the pilgrims are considered as committing a sin. Wajib 'Umrah consist of two specifically niyyah at Miqat and avoid to do some activities that prohibited during Ihram.

\subsection{Virtual Reality (VR) Technology}

Virtual Reality is a significant component that can make Virtual 'Umrah application successful. Virtual Reality technology is not fully utilized yet in any applications that deliver the knowledge of 'Umrah. Nevertheless, this technology is beneficial to the pilgrims when it is combined with multimedia elements in 'Umrah applications.

Virtual Reality technology has started since 1980[6] and was first introduced by Jaron Lanier. He was the pioneer and research group leader in virtual reality area. According to [7], Virtual Reality is a computer-based interface that consists of human perception and system reaction that use technology to adapt the situation, similar to the real world. Furthermore, Virtual Reality technology can improve the productivity in order to assist in visualizes the concepts, innovative and creative ideas [8]. Therefore, it can be concluded that Virtual Reality technology can be 
defined as a computer-based interface that is simulated by the use of virtual computer to let the users experience something that similar to the real world as well as the option to visualize, manipulate and interact with the system.

Virtual Reality system can be categorized into immersive, non-immersive and augmented reality [9]. Immersive can be defined as a system that requires special tools such as gloves, suit, and sophisticated computer to explore system development like stereoscopic, head mounted display (HMD), and large screen monitor. Whereas, non-immersive works at window based-system that require users to interact through computer's screen. The users can interact with the system via keyboard, mouse, joystick and touch screen. Virtual 'Umrah application is categorized as a nonimmersive virtual reality system as the users can look through the computer and directly interact with the system using the mouse, touchpad or the touch screen.

There are three types of modeling techniques in Virtual Reality technology namely geometric based model, image based model and hybrid based model [8]. Geometric model is based on geometric source. The resources in Geometric model are designed by using multimedia software. In contrast, image model resources are designed from the original image while hybrid model is a combination between geometric model and image model.

Image based model is adopted as modeling techniques for Virtual 'Umrah application. According to [10] the use of image is very useful in Virtual Reality environment. There are several advantages of image model such as better quality image and faster rendering process compared to geometric model and hybrid model. Moreover, image model can provide panoramic environment interactively with higher sense of presence and the users can feel real experience in performing 'Umrah rituals such as tawaf and tahallul (cutting of hair or shaving). The uses of Virtual Reality technology can be an alternative method to deliver knowledge to the 'Umrah pilgrims. Usually they get the information through conventional methods such as books, presentation slides and verbally by the religious teacher. This technology is proven can create an informal learning process to users because it can be done anytime, everywhere and this learning process will occur continuously as long as being used by the users [11]. Moreover, with the existing of continuous learning process, the users will get benefit from this application since they will get clearer understanding on how to perform 'Umrah.

\subsection{Multimedia Elements}

One of the components in Virtual 'Umrah application is multimedia elements. Multimedia elements can be described as different types of information in a multimedia presentation, including text, images, video, audio and animations.

\subsubsection{Text}

In any materials that assist the education process, the common element of multimedia that can be found is text. Text is a few words appearing in a predominantly graphic multimedia system that can have a powerful effect. Text also can be powerfully suggestive, engaging a reader's imagination, creating its own image, or prompting a string of unexpected reflection. In Virtual 'Umrah application, text is used in every page to explain the images, videos and others multimedia elements. Text also applied in menu that direct user to others page [12].

\subsubsection{Image}

According to [13], image can be described as digital representation of non-text information such as a drawing, chart or photo. Image can be categorized into two types namely vector graphic and 
The International Journal of Multimedia \& Its Applications (IJMA) Vol.5, No.5, October 2013

bitmap. Vector graphic can be resized without reducing the quality of the image in contrast bitmap can reduce the quality if the image is resized. Both categories are used in the development of Virtual 'Umrah and most of the images is originally hand drawn and reflected to Muslim way of life.

\subsubsection{Video}

Video is composed of a series of rapidly displayed still image. There are two types of video; analog and digital video. Digital video are used in the development of Virtual 'Umrah application. According to [14], video technologies have the abilities to capture the rich contexts of teaching and learning, with the necessary perspective to observe and reflect. Video element in Virtual 'Umrah is used to replace the text as a tool to explain some of the contents which is hard to explain by words and to show real practice for certain tasks in performing 'Umrah. The users can clearly see how that practical aspect has been carried out instead of they read the text without knowing how it is being done which can contribute to better understanding of that subject matters.

\subsubsection{Audio}

Audio is another element that can be used in Virtual 'Umrah application. Audio is suitable for this application because it can provide better quality of communication between user interface and the user [15]. When the quality is better, the user can gain information easily and smoothly. For this application, the audio is being captured via recording the actor's voice. The recorded voice is then processed using the sound editing software.

\subsubsection{Animation}

According to[16] a lot of information can be transferred by using moving image called animation because the eye-brains assembles a sequences of image and interprets them as a continuous movement. Animation can be defined as rapid display of images in sequence to create an impression of movement where it incorporates and draws inspiration from each of the other media [12]. In Virtual 'Umrah, animation can be used to help pilgrims learn more on how to perform 'Umrah because sometimes expressing ideas with texts or pictures only are not enough to make users more engage with the application.

\subsection{User Profile}

User-centered design (UCD) approach is a method that actively involves users at every stage of the design and development process. The goal of UCD is to ensure that a product fulfils the needs and requirements of the intended users. To enable to produce an effective and efficient designs are mainly influenced by the user's attitudes, abilities, skills and experiences to acquire and process information [17].

This section focuses on discussion that related to user skill levels and profiling tasks and user needs according to Virtual 'Umrah requirements. User characteristics capture the key attributes of the intended user group and these attributes affects the ways in which interaction is designed. The attributes that involve in this application are expertise, frequency, age, gender, educational background, computer/IT experience and motivation. For this application, the attributes such as expertise, frequency, education and computer experience are important because this application has interactive and multimedia elements that need skills and experience from the users to use [17]. 
The International Journal of Multimedia \& Its Applications (IJMA) Vol.5, No.5, October 2013

A successful design and development of a product has a lot to do with recognizing and understanding the users. Generally users can be categorized into three main category namely novice users, casual users and expert users. The first user is novice users or known as new or inexperienced users. These users require extensive guides in implementing their tasks for example by providing instruction in every task. They also need more responses and closure to enable users to know their progress when using the system. Casual users can be defined as user that able to complete their task without much help from the system. These users need a consistent structure to enable users to make predictions about the system and also possess efficient help facilities and documentations to remind them when they have forgotten information about the system. Lastly expert users are type of user that rarely need guidance and the system should facilitate methods that can implement tasks as quickly as possible with minimum error [17].

Eight golden rules by [17] are rules that usually an interactive system such as Virtual 'Umrah follows to design user interface. The rules are:

a) To be consistent in every aspects and actions such as colour, fonts, layout and so on.

b) Ensure that all intended users such as novice, casual and expert users can use the same interface.

c) Make sure that every action would be followed by appropriate feedback.

d) Design some sort of closure for example a dialog that display 'Thank you' after a transaction process is finished.

e) Should create some mechanisms to detect and prevent or correct errors.

f) Ensure that some actions can be reversed to prevent the users from being annoyed.

g) Support users with offering controls and freedom so that they have fun when using our application.

h) Decrease short term memory to avoid users to remember a lot of information when using the interface.

The design of user interface interrelated with user profile as mentioned before. A successful user interface in Virtual Reality environment has to accommodate the needs of every users either novice users, casual users or expert users. According to [18] a certain guidelines can be adopted to design an interface that includes:

a) The representation of anything from the real world should be natural or real.

b) Interaction techniques should be chosen carefully so that it will efficiently and effectively working in virtual environment.

c) Provide more responses and closure to enable users to know their progress when using the application.

\subsection{Usability Evaluation}

Usability is a concept in Human Computer Interaction (HCI) that refers to create any computer applications that easy to learn and easy to use through User-centered Design (UCD) process [19] [20]. UCD is considered especially important when creating new applications that need to be accepted by users.

According to [21] the usability concept consists of five main criteria namely easy to learn, easy to use, easy to remember, contain few errors and satisfaction. Meanwhile, [22] describe the usability criteria as effectiveness, efficiency, safety, utility, learnability and memorability. For this research, the authors have chosen usability criteria as learnability, efficiency, memorability, errors and satisfaction. Table 1 shows the definition of those criteria. 
The International Journal of Multimedia \& Its Applications (IJMA) Vol.5, No.5, October 2013

Table 1. Usability criteria of Virtual 'Umrah.

\begin{tabular}{|l|l|}
\hline \multicolumn{2}{|c|}{ Usability Criteria } \\
\hline Learnability & Users must be able to learn easily even after a period of not having used it. \\
\hline Efficiency & Users can carry out their tasks with a minimal number of steps. \\
\hline Effectiveness & Users are able to complete the task and to achieve goals. \\
\hline Errors & $\begin{array}{l}\text { Users can overcome any errors by providing button for help and display } \\
\text { suggestion through dialog box. }\end{array}$ \\
\hline Satisfaction & Users must be fun to use the system to feel satisfaction \\
\hline
\end{tabular}

Usability is important feature in order to evaluate an application [23]. The results from the usability evaluation can be used to judge the application. The term evaluation generally refers to the process of gathering data about the usability of a design or product by a specified group of users for a particular activity within a specified environment. In term of Virtual 'Umrah application, usability evaluation is a technique to identify user requirements and propose suitable solutions.

Before perform the evaluation, the authors determined the objectives of the evaluation. The planning for the evaluation is important in order to perform the evaluation smoothly. The objectives that have been identified for this research are as followed:

a) To ensure that users easy to learn the functions of tool at the interface in order to offer smooth interaction. (Learnability)

b) To make sure users can do their task efficiently by using help and also use hint for the button, then users can click in order to get more information. (Efficiently)

c) To assured users can adequate to remember the flow of the content or page navigation. (Memorability)

d) To ascertain users can explore the application with help button and dialogue box that can help them to settle the errors. (Errors)

e) To make sure users get the required information quickly and accurately as well as a high level of interactivity. (Satisfaction)

In order to perform usability evaluation, there are a few techniques that can be used which comprise of heuristic evaluation, performance measurement, think aloud protocol, observations, questionnaires, interviews, focus groups, use the actual logging and user response. Table 2 shows the usability techniques that will be implemented in this research.

Table 2. Usability techniques in Virtual 'Umrah

\begin{tabular}{|l|l|c|c|c|c|}
\hline \multirow{2}{*}{ No. } & \multirow{2}{*}{ Technique } & \multicolumn{4}{|c|}{ Phases } \\
\cline { 3 - 6 } & & Analysis & Design & Dev. & Eva. \\
\hline 1 & Observation & $\mathrm{X}$ & & & \\
\hline 2 & Questionnaire & $\mathrm{X}$ & & & $\mathrm{X}$ \\
\hline 3 & Interview & $\mathrm{X}$ & & & \\
\hline 4 & $\begin{array}{l}\text { Think aloud } \\
\text { protocol }\end{array}$ & & $\mathrm{X}$ & & \\
\hline 5 & User feedback & $\mathrm{X}$ & $\mathrm{X}$ & $\mathrm{X}$ & $\mathrm{X}$ \\
\hline 6 & Focus group & & & & $\mathrm{X}$ \\
\hline
\end{tabular}


The International Journal of Multimedia \& Its Applications (IJMA) Vol.5, No.5, October 2013

The implementation of these techniques is depending on the appropriateness of intended research. In Virtual 'Umrah application, the techniques to perform usability evaluation are think aloud protocol, observation, questionnaire, interview, focus group and feedback users [24].

a) Observation: Observation technique is the way of gathering data by watching both behaviors and physical characteristics of users in their natural setting. In analysis phase of Virtual 'Umrah development, observation technique is used to identify existed problems. During observation, the authors observe websites that contain an interactive application pertaining 'Umrah.

b) Questionnaire: Questionnaire technique is applied in analysis phase to get pilgrims' requirements as preliminary study. A pilot study is carried out to ensure the reliability of this instrument. For Virtual 'Umrah application, the questionnaire use Likert scale that range between 1 and 5. According to Nielson (1993) criteria for subject must indicate the average value of at least 4.An Alpha Cronbach coefficient can interpret the correlation coefficient with the difference in value between 1 and 0 [25]. This coefficient is used to determine the reliability of the questionnaire items. According to [26] and [27] the appropriate reliability coefficient is greater than 0.70 .

c) Interview: The authors use interview technique in order to acquire contents for Virtual 'Umrah application. It involves interviewer ask various questions to obtain the necessary information. The interviewees that involve in this interview are the experts in 'Umrah activities and rituals and usually the persons who are involved directly with the tourism agencies.

d) Think aloud protocol: This technique is used in the design phase to get visualization of the interface. Users involve directly providing ideas and concepts of interface design for the interactive application of 'Umrah. These activities are performed repeatedly until the user requirements are achieved.

e) User feedback: User feedback technique is implemented through the whole phases of developing Virtual 'Umrah application. User feedback is important because this application adopts UCD methodology that requires users' response iteratively for entire development process until the intended goals are achieved.

f) Focus group: In the evaluation phase, this technique is used to compare the usability of the proposed applications namely Virtual 'Umrah with the existing applications that use traditional approach.

There are numerous benefits of usability evaluation such as developers can save time to finish the project and also can save money. Besides that, people can enhance the creativity of the design whether in terms of interface or interaction. According to [28] the beneficiaries of usability are both the users and companies of the products. The overall advantages of usability include increased productivity, decreased costs of development and support, increased return on investment and increased user satisfaction.

\section{Conclusions}

The present paper reviews work in virtual environment specifically in 'Umrah. The components that important in virtual environment are also discussed namely content, virtual reality (VR) technology, multimedia elements, user profile and usability evaluation. Content is an important component that must be considered when developing Virtual 'Umrah because the contents will be able to provide accurate and precise information on rituals and activities in pertaining 'Umrah. Meanwhile, VR technology can be defined as a computer-based interface that is simulated by the use of virtual computer to let the users experience something that similar to the real world as well as the option to visualize, manipulate and interact with the application. Multimedia elements can be described as different types of information in a multimedia presentation, including text, images, video, audio and animations. These multimedia elements are used in every module in the 
The International Journal of Multimedia \& Its Applications (IJMA) Vol.5, No.5, October 2013

application based on user requirements. User profile focuses on discussion that related to user skill levels and profiling tasks and user needs according to UCD model that focuses on involvement of users in every phase. Lastly in term of Virtual 'Umrah application, usability evaluation is a technique to identify user requirements and propose suitable solutions. These components are hopefully can be implemented as guidelines to the others to develop a virtual environment. These components is hopefully can be implemented as guidelines to the others to develop the virtual environment.

\section{ACKNOWLEDGEMENTS}

The authors wish to thank to Muhammad Safwan Bin Mat Noh for his contribution to this research. The authors also would like to thank all colleagues who have been giving support and encouragement throughout the implementation of this study.

\section{REFERENCES}

[1] Hameed, S. A. (2010). ICT to serve Hajj: Analytical study. International Conference on Computer and Communication Engineering (ICCCE'10), (May), 1-7. IEEE.

[2] M.Fitri,Y,A, Nasir, Z, Norfadziana, F.M. (2011). Virtual Hajj (V-Hajj). IEEE Conference on Open System (ICOS2011), September 25-28, Langkawi, Malaysia.

[3] Yasin, A. M., Darleena, Z., \& Isa, M. A. M. (2012). Avatar Implementation in Virtual Reality Environment using Situated Learning for "Tawaf". Procedia-Social and Behavioral Sciences, 67, 7380.

[4] Al-Aama, A. (2008). A Proposed Framework for the Development of Web-Based Systems for the Service of Muslim Pilgrims. Science, 20(2), 55-75.

[5] Jamalluddin Hashim (2011). Panduan Mengerjakan 'Umrah. UniSZA.

[6] Oxford English Dictionary (OED)

[7] David R. Shanks, Marks F. St. John (1994). Characteristics of Dissociable Human Learning Systems. Behavioral and Brain Sciences, 17, 367-447.

[8] Wan Norazlinawati Wan Abd Arif, Wan Fatimah Wan Ahmad, Shahrina Md. Nordin, Azrai Abdullah \& Subarna Sivapalan. 2009. Designing 3 Dimensional Virtual Reality Using panoramic image.

[9] Whyte, J., (2002). Virtual Reality and the Built Environment. Architectural Press, Oxford.

[10] Sommerer, C., Mignonneau, L. \& Lopez-Gulliver, R. 1999. Time-lapse: an immersive interactive environment based on historic stereo images.

[11] Roussos, M. 1997. Issues in the Design and Evaluation of a Virtual Reality Learning Environment. Electrical Engineering and Computer Science. Chicago. University of Illinois at Chicago. Master of Science. 68.

[12] Savage, T. M. and Vogel, K. E. (2014). An Introduction to Digital Multimedia. 2nd Edition: Jones \& Bartlett Learning Publication.

[13] Shelly, G.B and Vermaat, M.E (2012). Discovering Computers: Your Interactive Guide to Digital World. Couse Technology.

[14] J. Wang and K. Hartley, Video Technology as a Support for Teacher Education Reform. Vol. 11 No. 1. (2003).

[15] Lopez, L.A. and Romer, R.M. (2010). New Perspectives on Adobe Flash Professional CS5, Comprehensive. Thomson Course Technology.

[16] Mohd Izani, Aishah', Ahmad Raifi Eshaq' and Norzaiha, Keyframe Animation and Motion Capture for Creating Animation: A Survey and Perception from Industry People, Student Conference on Research and Development (SCOReD) 2003 Proceedings, Putrajaya, Malaysia.

[17] Shneiderman, S. B., \& Plaisant, C. (2010). Designing the User Interface. 5th Edition.

[18] Bowman, D. A., Kruijff, E., LaViola Jr, J. J., \& Poupyrev, I. (2001). An introduction to 3-D user interface design. Presence: Teleoperators \& Virtual Environments, 10(1), 96-108.

[19] Lee, S. H. (1999). Usability testing for developing effective interactive multimedia software: Concepts, dimensions, and procedures. Educational Technology \& Society, 2 (2).

[20] Preece, J., Rogers, Y. \& Sharp, H. (1994). Interactions Design Beyond.

[21] Nielsen, J. (1993). Usability Engineering. Academic Press, Boston. 
The International Journal of Multimedia \& Its Applications (IJMA) Vol.5, No.5, October 2013

[22] Preece, J., Rogers, Y. \& Sharp, H. (2002). Interactions Design Beyond.

[23] Ardito, C., Costabile, M.F., De Marsico, M., Lanzilotti, R., Levialdi, S., Roselli, T., \& Rossano, V. (2006). An Approach to Usability Evaluation of e-Learning Applications. Universal Access in the Information Society International Journal, 4(3), 270-283.

[24] Nielsen, J. 2003. Usability 101: Introduction to usability. http://www.useit.com/alertbox/20030825.html.

[25] Coakes, S.J. (2005). SPSS 12.0 for windows analysis without Anguish.

[26] Pallant, J. 2001. SPSS survival Manual. A Step By step Guide to Data Analysis Using SPSS for Windows (Version 10). NSW: Allen\&Unwin.

[27] DeVellis, R.F. (2003). Scale development: Theory and applications. 2nd edition. Thousand Oaks, CA: Sage.

[28] Kaemba, M. (2008). Digital Museum Usability.

\section{AUTHORS}

Normala Rahim is a lecturer in University Sultan Zainal Abidin, Terengganu. In 2006, she holds a Diploma in Photography \& Creative Imaging from Universiti Teknologi Mara (UiTM). In 2008, she holds a Degree in Bachelor of Photography \& Creative Imaging (Hons) from Universiti Teknologi Mara (UiTM) and MIT (Information Science) from Universiti Kebangsaan Malaysia (UKM), Malaysia. Her research interests are Virtual Reality (VR) and Human Computer Interaction (HCI).

Nazirah Abd Hamid is a lecturer in University Sultan Zainal Abidin, Terengganu. She holds a degree in Bachelor of Information Technology from Universiti Utara Malaysia (UUM), in 2004 and M. Sc. Com. (Information Security) from Universiti Teknologi Malaysia (UTM), Malaysia. Her research interests are Information Security and Human Computer Interaction (HCI).

Wan Malini Wan Isa is a lecturer in Universiti Sultan Zainal Abidin, Terengganu. She holds a degree in Bachelor of Computer Science (Multimedia) from Universiti Putra Malaysia (UPM) in 2006 and M. Sc. Com. (Multimedia) from University Putra Malaysia (UPM), in 2008. Her research interests are Multimedia Technology and Human Computer Interaction (HCI).

Siti Dhalila Mohd Satar currently works as a lecturer at Faculty of Informatics and Computing, University Sultan Zainal Abidin, Terengganu. She holds a B.S. degree in Information Technology from Universiti Kebangsaan Malaysia (UKM), in 2008 and MSc in Computer Science (Information Security) from Universiti Teknologi Malaysia (UTM), Malaysia. Her research interests are Information Security, and Information Quality.

Azilawati Rozaimee is a lecturer in University Sultan Zainal Abidin, Terengganu. She holds a degree in Bachelor of Computer Science (Software Engineering) from Universiti Putra Malaysia (UPM) in 2006 and M.Sc.Com. (Software Engineering) from Universiti Putra Malaysia (UPM), Malaysia in 2008. Her research interests are Multimedia Technology and Human Computer Interaction (HCI).
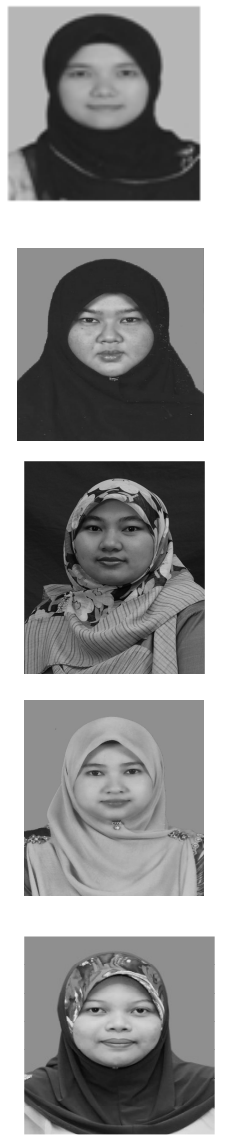